\title{
OPEN Value of liver iron concentration in healthy volunteers assessed by MRI
}

\author{
Marzanna Obrzut ${ }^{1,2}{ }^{2}$ Vitaliy Atamaniuk ${ }^{1 凶}$, Kevin J. Glaser ${ }^{2}$, Jun Chen ${ }^{2}$, Richard L. Ehman ${ }^{2}$, \\ Bogdan Obrzut ${ }^{3}$, Marian Cholewa ${ }^{1}$ \& Krzysztof Gutkowski ${ }^{4}$
}

Iron overload is a relatively common clinical condition resulting from disorders such as hereditary hemochromatosis, thalassemia, sickle cell disease, and myelodysplasia that can lead to progressive fibrosis and eventually cirrhosis of the liver. Therefore, it is essential to recognize the disease process at the earliest stage. Liver biopsy is the reference test for the assessment of liver fibrosis. It also allows for quantifying liver iron concentration (LIC) in patients. However, this is an invasive method with significant limitations and possible risks. Magnetic resonance imaging (MRI) and evaluation of the R2* relaxation rate can be an alternative to biopsy for assessing LIC. However, it causes a need for accurate R2* data corresponding to standard value for further comparison with examined patients. This study aimed to assess the normative values of liver $\mathrm{R} 2$ * in healthy individuals. A total of 100 volunteers that met established criteria were enrolled in the study: 36 (36\%) men and 64 (64\%) women. The mean age was 22.9 years (range 20 to 32 years). R2* was estimated by an MRI exam with a $1.5 \mathrm{~T}$ clinical magnetic resonance scanner. Images for measuring the LIC and liver fat concentration were obtained using the IDEAL-IO technique for liver imaging. The Mean (SD) liver R2* was $28.34(2.25) \mathrm{s}^{-1}(95 \% \mathrm{Cl}, 27.78-$ 28.90, range 23.67-33.00 s $\left.\mathrm{s}^{-1}\right)$ in females, $29.57(3.20) \mathrm{s}^{-1}\left(95 \% \mathrm{Cl}, 28.49-30.66\right.$, range 23.93-37.77 $\left.\mathrm{s}^{-1}\right)$ in males, and $28.72(2.69) \mathrm{s}^{-1}$ (range 23.67-37.77 $\mathrm{s}^{-1}$ ) in the whole group. R2* value in this particular population with a high proportion of young women did not exceed $38 \mathrm{~s}^{-1}$. In the absence of fibrosis or steatosis, liver stiffness and fat fraction did not show any relationship with R2*.

Iron overload is a relatively common clinical condition, resulting from disorders such as hereditary hemochromatosis, thalassemia, sickle cell disease, and myelodysplasia ${ }^{1}$. Iron accumulation can result from excessive iron supply or absorption. The most common cause of excessive iron supply is secondary to red blood cell (RBC) transfusions used to treat chronic anemia (e.g., thalassemia, sickle cell disease, inherited bone marrow failure syndrome, and myelodysplastic syndrome). A less common cause is the use of supplements or therapeutic infusions rich in iron, such as hemin used to treat some types of porphyria.

The most common cause of increased iron absorption in adults is congenital hemochromatosis caused by gene mutation, inefficient erythropoiesis in the course of thalassemia, sideroblastic and other hereditary anemias, and liver diseases (in particular, acute liver disease (ALD) and chronic diseases like nonalcoholic fatty liver disease (NAFLD) and chronic viral hepatitis). Usually, patients have more than one reason for iron overload. The mechanism for iron absorption in the liver is not fully understood and is most likely associated with a decrease in hepcidin production in the diseased liver ${ }^{2}$.

Irrespective of the causes of iron overload, the liver is the organ that is most affected by iron overload. In the case of increased intestinal iron absorption due to the accumulation and damage of hepatocytes, this can lead to progressive fibrosis and eventually cirrhosis of the liver, resulting in portal hypertension, liver failure, and hepatocellular carcinoma ${ }^{3,4}$. Therefore, it is extremely important to recognize the disease process in as early a stage as possible. Liver biopsy is considered to be the definitive test for the assessment of liver fibrosis. In the setting of iron overload, a biopsy can be used to quantify liver iron concentration (LIC) $)^{5}$. However, this is an invasive method with substantial sampling error. A significant limitation of this diagnostic method is the fact,

\footnotetext{
${ }^{1}$ Department of Biophysics, College of Natural Sciences, Institute of Physics, University of Rzeszow, Rzeszow, Poland. ${ }^{2}$ Department of Radiology, Mayo Clinic, Rochester, MN, USA. ${ }^{3}$ Department of Obstetrics and Gynecology, Institute of Medical Sciences, Medical College, University of Rzeszow, Rzeszow, Poland. ${ }^{4}$ Department of Gastroenterology and Hepatology with Internal Disease Unit, Teaching Hospital No. 1, Rzeszow, Poland. ${ }^{\square}$ email: vitaliy.atamaniuk@gmail.com
} 
that the distribution of iron in the liver is inherently very heterogeneous ${ }^{6}$. Besides, this method carries a risk of complications, such as postinterventional hemorrhage and bleeding ${ }^{7,8}$.

Due to the known systematic and accidental errors, costs, and invasiveness, a biopsy cannot be considered a gold standard for LIC assessment and should not be used for diagnostics except for disease processes requiring histological assessment of the organ to establish a diagnosis and correct treatment ${ }^{6}$.

Magnetic resonance imaging (MRI) techniques have been advocated as an alternative to biopsy for assessing LIC. One approach is to measure the $\mathrm{R} 2^{*}$ relaxation rate of liver tissue by using a multipoint Dixon sequence such as IDEAL-IQ ${ }^{9}$. Studies have shown that R2* increases systematically with hepatic iron concentration ${ }^{10}$. This parameter has a high correlation with biopsy measurements and could be a reliable, noninvasive method for measuring $\mathrm{LIC}^{10}$. For MRI to be used for the rapid routine assessment of LIC, it is necessary to establish ranges of $\mathrm{R} 2{ }^{*}$ values for the healthy population. To our knowledge, there is little data in the literature assessing the liver iron level in healthy adult volunteers ${ }^{11}$. Published studies have qualified the absence of liver disease in cohorts by the participant's declaration of the absence of liver disease without the confirmation of this fact, even using basic laboratory tests. There is a need to obtain objective R2* data based on representative groups of healthy subjects who undergo more tests to confirm their health status, especially to confirm their lack of liver inflammation and fibrosis.

\section{Objectives}

To assess the normative values of liver R2* measured by the IDEAL-IQ MRI sequence in healthy volunteers.

Study enrollment. 115 subjects were primarily enrolled in the study. The inclusion criteria were as follows: no contraindications to MRI, no history of liver disease, no risk factors for chronic liver disease, negative family history of chronic liver disease, normal liver enzyme levels, normal ferritin (13-150 ng/ml for women, $30-400 \mathrm{ng} / \mathrm{ml}$ for men) and iron values (37-170 mcg/dl for women, $49-181 \mathrm{mcg} / \mathrm{dl}$ for men), no drug or medication use of any kind and absence of liver fibrosis or steatosis on MRI. As exclusion criteria were used following values of parameters: liver stiffness $<2.9 \mathrm{kPa}$ and fat fraction $<5 \%{ }^{12}$. All subjects declared they consumed less than $30 \mathrm{~g}$ of alcohol per day. The participants were not required to change their normal diet to be included in this study but required $6 \mathrm{~h}$ of fasting before the MRI scans. The research was carried out in the magnetic resonance laboratory of the Center for Medical and Natural Sciences Research and Innovation of the University of Rzeszow, Poland in the period from December 2018 until July 2019.

Study protocol. Subjects were examined on a 1.5-T whole-body OPTIMA MR360 Advance MR scanner (GE Healthcare, Milwaukee, Wisconsin, United States) with software version SV23. This device is equipped with a gradient system with a maximum amplitude of $33 \mathrm{mT} / \mathrm{m}$ and a $120 \mathrm{mT} / \mathrm{m} / \mathrm{ms}$ slew rate. For liver MRI and MRE examinations, all patients underwent the scan in the feet-first supine position with an 8-channel torso phased-array coil around the abdomen. As part of the MRE setup, a drum-like passive liver driver was secured to the right side of the chest wall with its center at the superior-inferior (SI) level of the xiphoid process. There was a 29-foot long PVC tube (3/4-inch diameter) connecting the passive driver to an active driver (the source of the vibrations used during MRE acquisitions) located in the adjacent equipment room.

Images for measuring the LIC and liver fat concentration were obtained using the IDEAL-IQ technique for liver imaging (GE Healthcare, Waukesha, WI, USA) and was performed using a gradient-echo multi-echo MR sequence with the following imaging parameters: $\mathrm{FOV}=39 \mathrm{~cm}, \mathrm{TR}=12.4 \mathrm{~ms}$, acquisition matrix $=160 \times 160$, slice thickness $=10 \mathrm{~mm}$, slice number $=28$. Six echo times were collected and $\mathrm{R} 2^{\star}$ and fat-fraction images were generated automatically on the scanner. The acquisition was performed in a single breath-hold of $17 \mathrm{~s}$.

Liver stiffness images were obtained using the MR-Touch MRE technique (GE Healthcare, Waukesha, WI, USA) using a 2D gradient-echo pulse sequence with a $60-\mathrm{Hz}$ motion frequency. The imaging parameters were as follows: $\mathrm{FOV}=40 \mathrm{~cm}, \mathrm{TR}=33.3 \mathrm{~ms}, \mathrm{TE}=20.5 \mathrm{~ms}$, acquisition matrix $=224 \times 64$, parallel imaging factor of 2, reconstructed matrix size $=256 \times 256$, slice thickness $=10 \mathrm{~mm}$, flip angle $=30^{\circ}$, number of time offsets $=4$, time of acquisition $=1 \mathrm{~min} 8 \mathrm{~s}$. Nine slices were obtained through the liver in three or four breath holds, and the subjects held their breath at the end of expiration. Images of the liver stiffness (elastograms) were generated automatically on the scanner and were transferred offline for additional analysis and measurements.

Image analysis. To measure liver $\mathrm{R} 2^{\star}$ and fat concentration, four irregular polygon regions of interest (ROIs) were defined in the liver on four consecutive $\mathrm{R} 2{ }^{*}$ images and were applied to the fat content images as well. ROIs were drawn to be as large as possible while excluding large vessels and the central biliary tree. Besides, the position of the ROIs was chosen in areas far from the organ boundaries. Liver R2* was calculated as the mean of the four R2* values and was used to estimate LIC according to Wood formula ([Fe] mg/g $\left.=\mathrm{R} 2^{\star} \times 0.0254+0.202\right)^{6}$. Since not all our patients had saved individual eight echoes in the clinic scans for calculating the offline classic $\mathrm{R} 2^{\star}$ results by using the magnitude-based monoexponential model ${ }^{6}$, we used the scanner-generated the complex-based IDEAL-IQ R2* results. A newly published study ${ }^{13}$ found the classic R2* and IDEAL-IQ R2* were significantly correlated to each other $(\mathrm{r}=0.9231, p<0.0001)$, and the patient $\mathrm{R} 2^{\star}$ range covered our patients $\mathrm{R} 2^{\star}$.

Liver stiffness was measured by placing a total of four irregular polygon ROIs in the right hepatic lobe on the elastograms, with large vessels and regions of low confidence excluded. Liver stiffness was calculated as the mean of the mean liver stiffness values obtained from each of the four middle slices, weighted by the ROI area.

Statistical analysis. Liver stiffness and liver $\mathrm{R} 2^{\star}$ values were presented as mean (SD). To assess the correlation of liver R2* with liver stiffness, liver fat fraction, and ferritin level, the Pearson correlation test was performed. The Mann-Whitney U test was used to identify differences between male and female volunteers. 


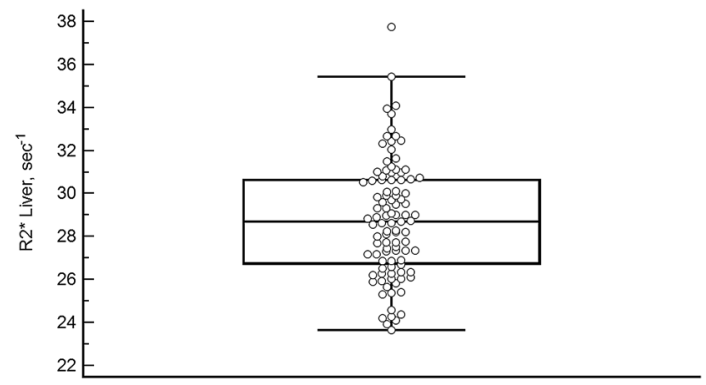

Figure 1. Liver $\mathrm{R} 2^{\star}$ values in normal volunteers. Box-and-whisker plot graph showing the distribution of the liver $2^{\star}$ values in the 100 normal healthy volunteers. Dots represent the value of each volunteer.

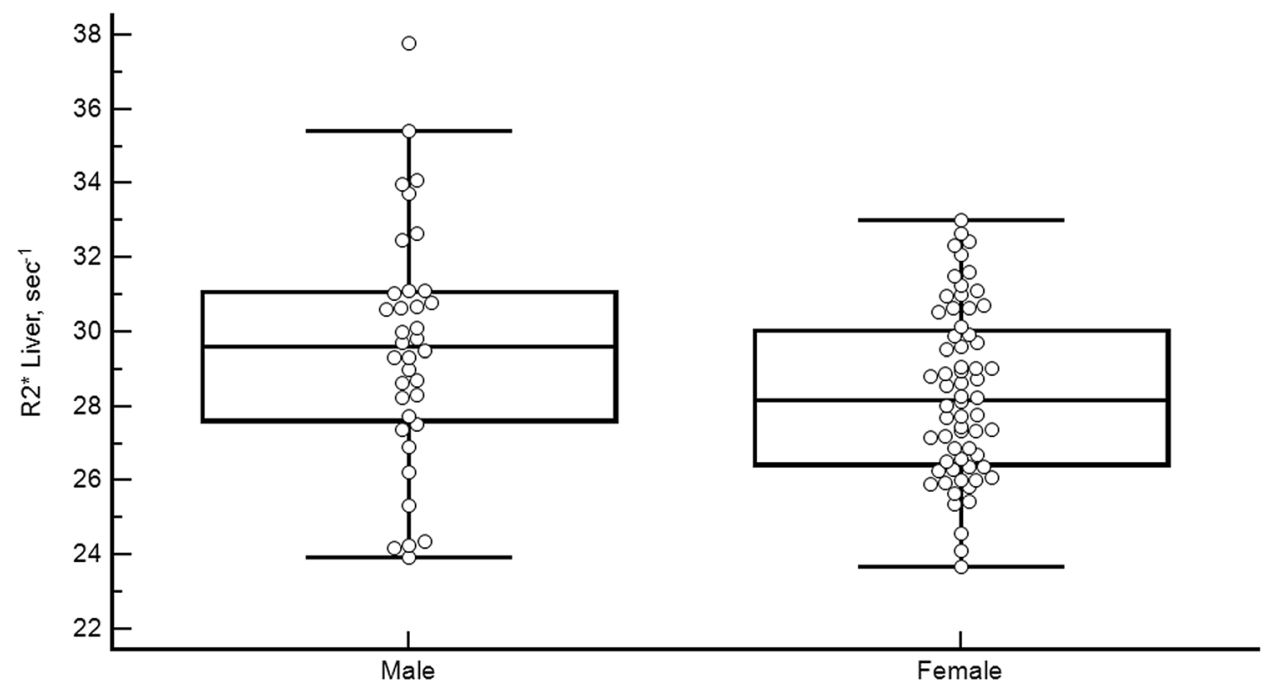

Figure 2. Liver $\mathrm{R} 2^{\star}$ values separated by sex. Boxplot graph showing the distribution of liver $\mathrm{R} 2{ }^{\star}$ values in the female and male volunteers.

Statistical significance was assumed at a $p$ value of less than 0.05. MedCalc software (version 18.9.1; Mariakerke, Belgium) was used to perform the statistical analysis.

Ethics. The study protocol was approved by the Ethics Committee of the Medical Department of Rzeszow University (Resolution No. 8/10/2016) and conformed to the ethical guidelines of the 1975 Declaration of Helsinki (6th revision, 2008). After receiving an explanation of the study, each subject provided written informed consent to enroll in the study.

\section{Results}

Of the 115 volunteers, 15 were excluded because of elevated (abnormal) values of aspartate aminotransferase (AspAT) and/or alanine aminotransferase (AlAT). The remaining 100 were considered eligible and were included in the study. All MRE exams were successfully completed. None of the volunteers reported any discomfort during the procedure. Of these 100 subjects, $36(36 \%)$ were male and $64(64 \%)$ were female and the mean age was 22.9 years (range 20 to 32 years). The mean BMI for whole group was $21.33 \mathrm{~kg} / \mathrm{m}^{2}$ (SD $2.19 \mathrm{~kg} / \mathrm{m}^{2}$, range: $16.85-25.76 \mathrm{~kg} / \mathrm{m}^{2}$. For female volunteers the mean BMI was $20.64 \mathrm{~kg} / \mathrm{m}^{2}$ (SD: $1.96 \mathrm{~kg} / \mathrm{m}^{2}$, range: $16.85-25.59 \mathrm{~kg}$ ) $\mathrm{m}^{2}$. For men, the mean BMI was $22.56 \mathrm{~kg} / \mathrm{m}^{2}$ (SD: $2.05 \mathrm{~kg} / \mathrm{m}^{2}$, range: $17.96-25.76 \mathrm{~kg} / \mathrm{m}^{2}$.

To measure liver R2*, the average size of the ROIs was 5 to $7 \mathrm{~cm}^{2}$ for the liver. The mean (SD) liver R2* for the population was $28.72(2.69) \mathrm{s}^{-1}\left(95 \% \mathrm{CI}, 28.26-29.32\right.$, range 23.67-37.77 s $\mathrm{s}^{-1}$ (Fig. 1). The hepatic iron load was assessed using the Wood formula $([\mathrm{Fe}] \mathrm{mg} / \mathrm{g}=\mathrm{R} 2 * \times 0.0254+0.202)$. This equates to a mean $\mathrm{LIC}$ of $0.93 \mathrm{mg} / \mathrm{g}$ (range $0.80-1.16 \mathrm{mg} / \mathrm{g})^{6}$.

Mean (SD) liver R2 ${ }^{\star}$ for the females was $28.34(2.25) \mathrm{s}^{-1}$ (95\% CI, 27.78-28.90, range 23.67-33.00 s${ }^{-1}$ ). For males, the mean was $29.57(3.20) \mathrm{s}^{-1}\left(95 \% \mathrm{CI}, 28.49-30.66\right.$, range 23.93-37.77 $\left.\mathrm{s}^{-1}\right)$ (Fig. 2). This difference was statistically significant $(p=0.04)$.

Figure 3 shows an example of an MRE scan. The liver stiffness in healthy volunteers ranged from 1.84 to $2.82 \mathrm{kPa}$, with a mean (SD) of $2.30(0.23) \mathrm{kPa}$ (95\% CI, 2.25-2.34). Mean (SD) liver stiffness for females was $2.31(0.23) \mathrm{kPa}(95 \% \mathrm{CI}, 2.26-2.37$, range $1.84-2.82 \mathrm{kPa})$ and for males was $2.27(0.23) \mathrm{kPa}(95 \% \mathrm{CI}, 2.19-2.35$, range $1.92-2.79 \mathrm{kPa})$. These values did not differ significantly $(p=0.35)$. There was no correlation between the 

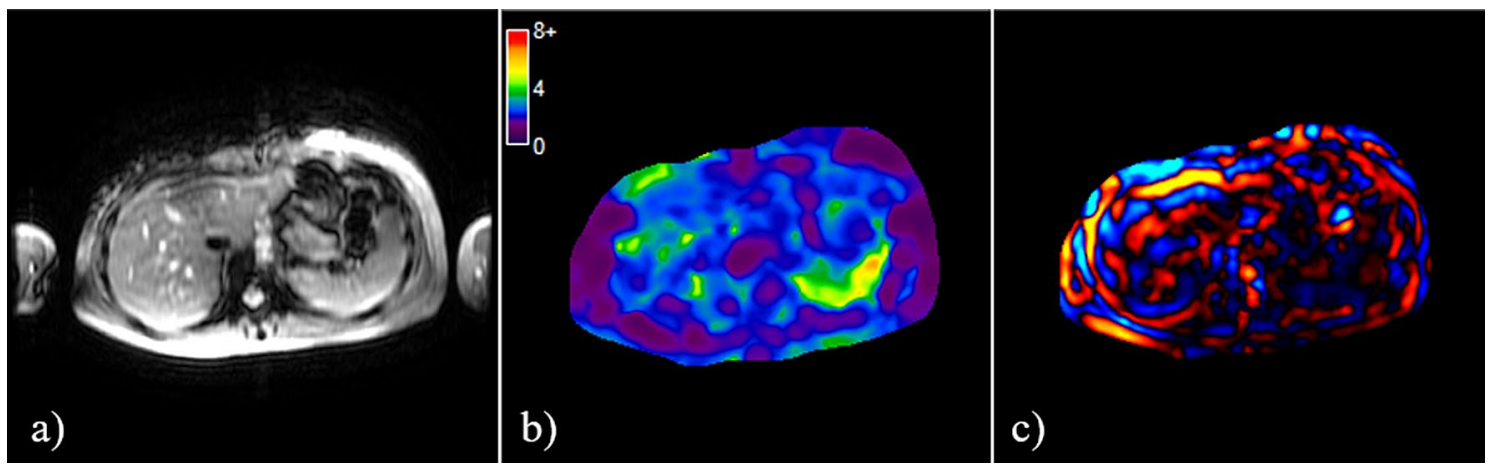

Figure 3. An example of MRE scan showing (a) magnitude, (b) elastogram, and (c) wave images.

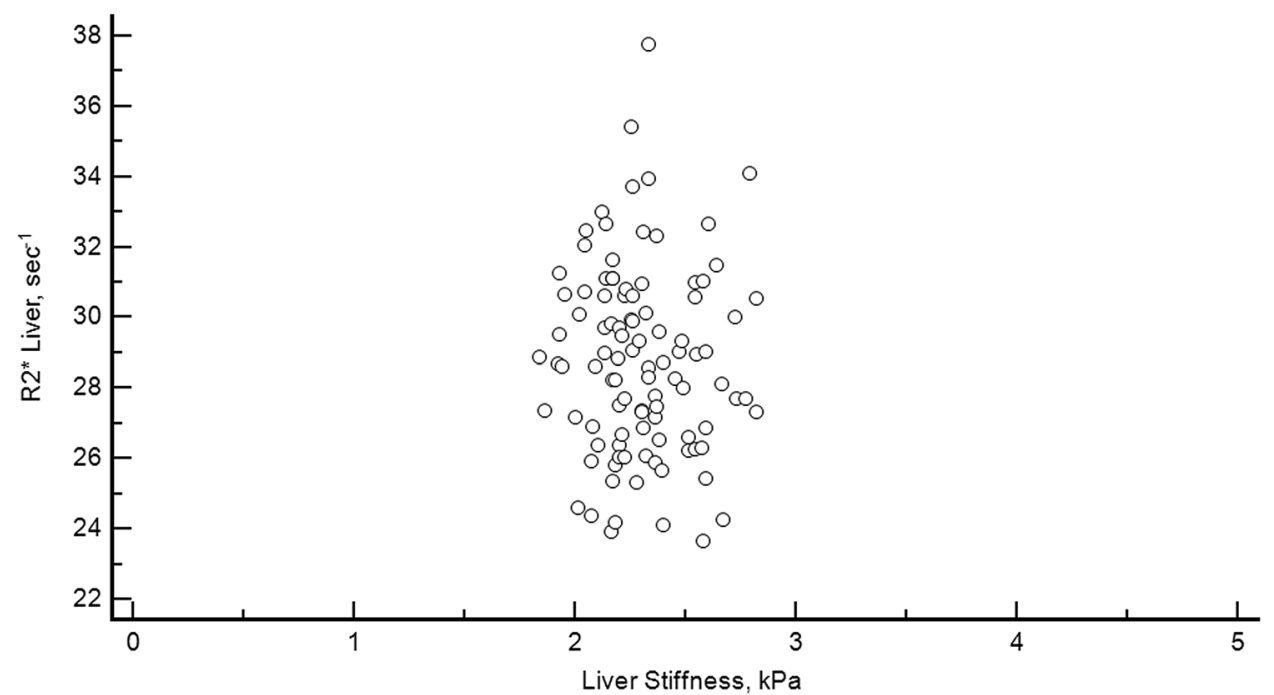

Figure 4. Relationship between liver stiffness and $\mathrm{R} 2^{\star}$. Scatterplot diagram showing the relation between liver stiffness and liver $\mathrm{R}^{*}$ in the 100 healthy volunteers.

liver stiffness and gender $(p=0.19)$. There was also no correlation between liver R2 ${ }^{\star}$ and liver stiffness $(\mathrm{r}=-0.04$, $p=0.68)$ (Fig. 3).

Liver fat fraction ranged from 1.40 to $4.90 \%$. Mean value (SD) was 2.51 (0.75)\% (95\% CI, 2.36-2.66\%). For males, the mean was $2.69(0.88) \%(95 \%$ CI, 2.39-2.99\%; range 1.70-4.90\%). For females, the mean was 2.41 (0.66) \% (95\% CI, 2.25-2.58\%; range 1.40-4.87\%). There was no correlation between the liver fat fraction and gender $(p=0.19)$. Liver $\mathrm{R} 2 *$ did not correlate with liver fat fraction $(\mathrm{r}=0.13, p=0.20)$.

Ferritin level ranged from 3.74 to $260.10 \mathrm{ng} / \mathrm{ml}$. Mean value (SD) was $53.29(52.15) \mathrm{ng} / \mathrm{ml}(95 \%$ CI, 42.94-63.64 ng/ml). For males, the mean was 93.31 (64.56) $\mathrm{ng} / \mathrm{ml}$ (95\% CI, 71.46-115.16 ng/ml; range $6.22-260.10 \mathrm{ng} / \mathrm{ml}$ ). For females, the mean was $30.78(22.98) \mathrm{ng} / \mathrm{ml}$ (95\% CI, 25.04-36.52 $\mathrm{ng} / \mathrm{ml}$; range $3.74-113.1 \mathrm{ng} / \mathrm{ml})$. This difference was statistically significant $(p<0.0001)$.

There was no correlation between liver $\mathrm{R}^{*}$ and ferritin level neither for males: $\mathrm{r}=0.28, p=0.096$ (Fig. 4), nor for females: $\mathrm{r}=-0.01, p=0.91$ (Fig. 5).

\section{Discussion}

The normal iron content in the human body is $40 \mathrm{mg} / \mathrm{kg}$ in women by menopause and $50 \mathrm{mg} / \mathrm{kg}$ in men ${ }^{14,15}$. About $80 \%$ of total iron in the human body occurs in the red blood cells and in proteins other than hemoglobin. The remaining $20 \%$ is largely stored in the ferritin and hemosiderin ${ }^{16,17}$. Ferritin acts as a deposit storing excess iron and occurs primarily in the liver, spleen, and bone marrow ${ }^{17}$.

A smaller amount of iron is associated with transferrin in plasma ${ }^{18}$. In women, the amount of stored iron is lower and additionally depends on the severity of menstruation, pregnancy, lactation as well as the amount of iron supplements taken. Premenopausal women lose iron due to menstruation at a rate of about $0.5-1.0 \mathrm{mg} / \mathrm{day}$.

There are no physiological mechanisms to eliminate iron from the body when there is excess ${ }^{2}$. If the supply of iron from the various sources is too high, then systemic iron overload occurs in the body. Initially, ferritin and hemosiderin (which is a partially denatured form of ferritin) store the excess iron. If the iron-binding capacity of the substances is exceeded, free iron is deposited in the cells of other organs. 


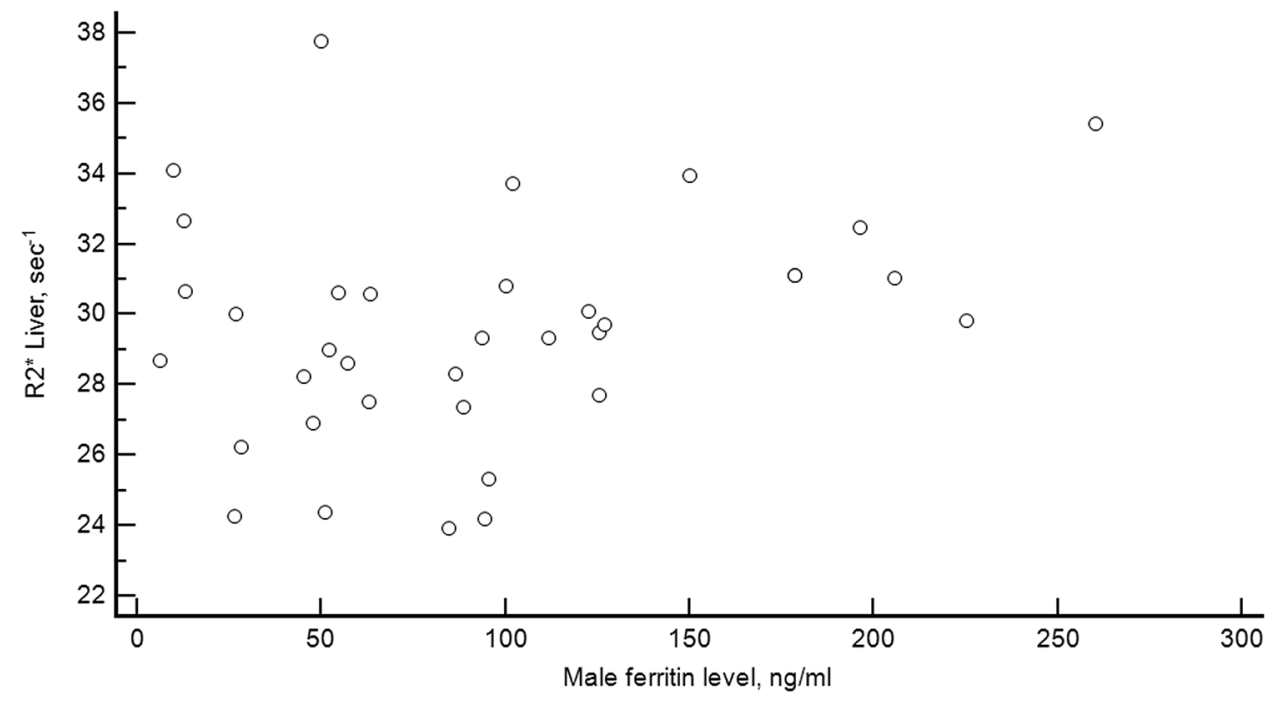

Figure 5. Relationship between ferritin level and liver R2* for males. Scatterplot diagram showing the relation between ferritin level and liver $\mathrm{R} 2 *$ in the 36 healthy male volunteers.

Even if the level of ferritin in the blood can be used as a rough indicator of liver iron overload in a highly overloaded population ${ }^{19}$, it is considered that it does not allow the diagnosis of liver iron overload and should not be used solely for clinical decision making ${ }^{20-22}$.

In the past 20 more years, many MRI methods have been developed to measure hepatic iron concentration, including SIR methods at $0.5 \mathrm{~T}^{23}, 1.5 \mathrm{~T}^{24,25}$, and $3 \mathrm{~T}^{26}, \mathrm{R} 2$ calculation at $1.5 \mathrm{~T}^{1}$ and $\mathrm{R} 2^{*}$ calculations at $1.5 \mathrm{~T}^{6,21,27-29}$ and at $3 \mathrm{~T}^{30}$.

Due to whole body iron metabolism ${ }^{31}$, MRI is the most practical and accurate method to monitor the concentration of iron in various human organs. The literature describes attempts to assess iron content in the body using $\mathrm{R} 2^{*}$ or $\mathrm{T} 2^{*}$ magnetization relaxation values $\left(\mathrm{R} 2^{*}=1000 / \mathrm{T} 2^{*}\right)$. The majority of the studies showed a good correlation between iron concentration in the liver and $\mathrm{T} 2$ or T2* in patients with iron-overload diseases in the course of congenital hemochromatosis, thalassemia, and sickle cell disease $\mathrm{e}^{1,6,19,20,23,25,32-37}$.

The MRI measurements hepatic $\mathrm{R} 2^{\star}$ or $\mathrm{T} 2^{\star}$ is a fast, widely available, and relatively inexpensive technique that some institutions are willing to use as an alternative to liver biopsies. However, for studies of T2*/R $2^{\star}$ in healthy individuals, the literatures only reported a small number of participants ${ }^{6,35}$. Maris et al. measured the T2* value for the liver in 21 healthy volunteers ${ }^{35}$. In the Wood study, for comparative purposes, data were obtained in a 13-person control group ${ }^{6}$. The only more notable work assessing the levels of $\mathrm{T}^{*}$ in the liver and spleen in healthy volunteers and comparing these values with the level of ferritin in blood ${ }^{11}$ is of limited value due to the non-objective criterion of qualification: only a participant health declaration without basic corroborating laboratory tests.

The results from our study fill this existing gap in the literature. In our work, we included healthy volunteers with no history of liver disease and negative blood tests that excluded inflammatory processes in the body. The absence of fibrosis processes in the liver was additionally confirmed by MR elastography exam. The stiffness of the liver was in the range of $1.84-2.82 \mathrm{kPa}$ with an average of $2.30(0.23) \mathrm{kPa}$, which was within the normal range ${ }^{38}$.

In our study, the average value of $\mathrm{R} 2^{*}$ was $28.751 \mathrm{~s}^{-1}\left(\mathrm{~T} 2^{*}=34.78 \mathrm{~ms}\right)$. For comparison, Anderson gives a $\mathrm{T} 2^{*}$ value of $33 \pm 7 \mathrm{~ms}$ for the liver and $56 \pm 22 \mathrm{~ms}$ for the spleen ${ }^{27}$. Maris et al. reported the average $\mathrm{T} 2^{\star}$ for the liver as $24.2 \pm 3.0 \mathrm{~ms}$ in 21 healthy volunteers ${ }^{35}$. The recommended upper limit of LIC is $<2 \mathrm{mg} \mathrm{Fe} / \mathrm{g}_{\text {dry liver tissue }}{ }^{39}$. Another study by Nuttall et al., based on a series of 141 liver biopsies, suggests reference limit $1.8 \mathrm{mg}$ Fe/g dry liver tissue in healthy adults ${ }^{40}$. The LIC increases with age, an average of $14 \mathrm{mcg} \mathrm{Fe} / \mathrm{g}$ per year ${ }^{40}$. As expected, the LIC values in women are lower than in $\mathrm{men}^{41}$. The above-mentioned LIC value of $2 \mathrm{mg} / \mathrm{g}$ is equivalent to the $\mathrm{R} 2^{*}$ value of about $70 \mathrm{~s}^{-1} 29$. The $\mathrm{R} 2^{*}$ value measured in our healthy volunteers corresponds well with the proposition of classification of iron overload severity offered by Henninger et al. ${ }^{39}$. In the 13 healthy volunteers ( 9 male, 4 female) with ages of $29.3 \pm 12.3$ years (range, $12-50$ years) in the Wood's study, the normal R2* was $39.9 \pm 2.8 \mathrm{~s}^{-1}(\mathrm{HIC}=1.17 \mathrm{mg} / \mathrm{g} \text { dry weight })^{6}$.

In the presented series, there was no correlation between $\mathrm{R} 2^{\star}$ and liver stiffness, neither for women nor for men. This is consistent with the results of other authors ${ }^{42}$.

Our study showed a clear difference in the mean liver R2* between men and women. Women had a statistically significantly lower R2 $2^{\star}$ value $\left(28.34 \mathrm{~s}^{-1}\right)$ compared to men $\left(29.57 \mathrm{~s}^{-1}\right), p=0.040$. This was expected as premenopausal women lose iron due to menstruation. However, premenopausal women lose iron due to menstruation so our women-dominated population of young healthy volunteers should has a lower iron level than a more general population that includes more elderly people and men.

Also, in this study, we only used the $\mathrm{R} 2^{*}$ images on the scanner, generated by the manufacturer IDEAL-IQ complex-based fat-water R2* model. There are many other magnitude-based R2* models, for example, Yokoo 


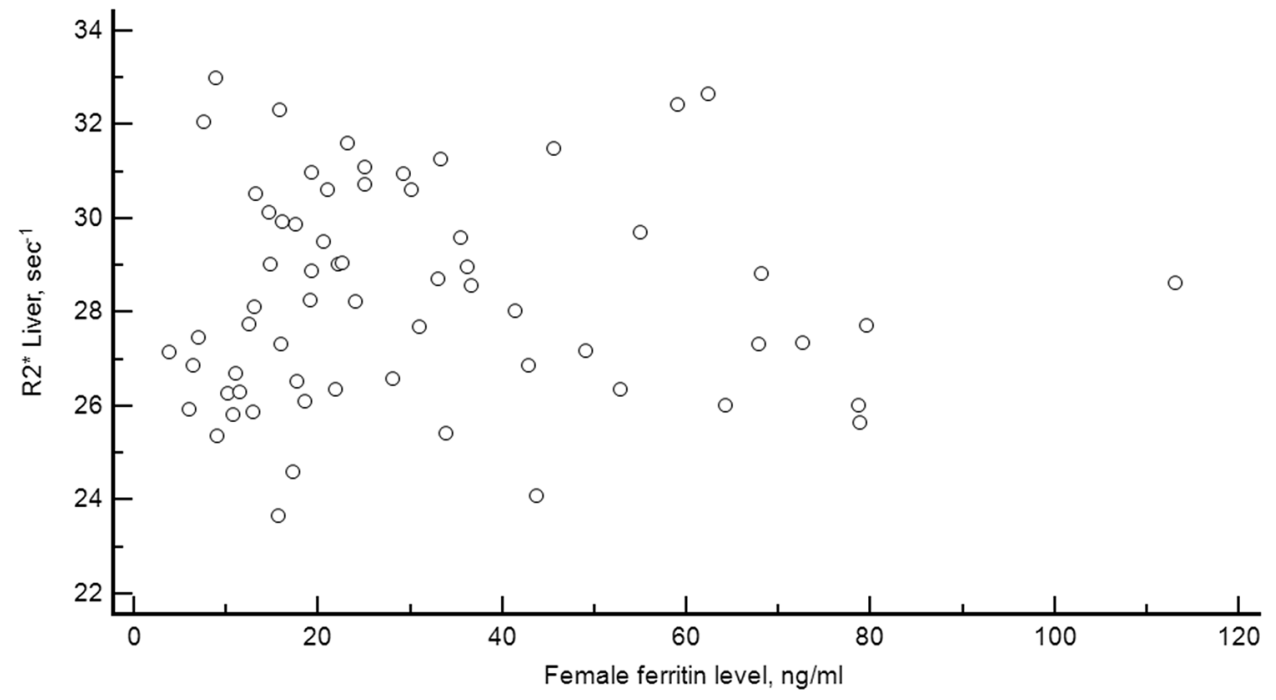

Figure 6. Relationship between ferritin level and liver R2* for females. Scatterplot diagram showing the relation between ferritin level and liver R2* in the 64 healthy female volunteers.

et al. ${ }^{43}$ studied the effect of Rician and non-Rician noise on the accuracy of R2* estimation in 7 different magnitude-based models, and found, the agreement of the R2* estimation was excellent in patients with low ferritin but poor in patients with high ferritin, as expected. Tipirneni-Sajja et al. ${ }^{44}$ simulated 5 different magnitude-based R2* models (GRE-A, GRE-B, GRE-C, UTE-A, and UTE-A), and found, the constant offset monoexponential model overestimated the $\mathrm{R} 2^{\star}$ at the lowest simulated SNR, and the monoexponential fitting with noise subtraction is the most robust and accurate $\mathrm{R} 2 *$ model for ultrashort TE (UTE) sequence.

A limitation of the presented work is the narrow age range of the studied group. Therefore, it was not possible to analyze the correlation of $\mathrm{R}^{*}$ with age. Further studies should include individuals within a wider age range to investigate such potential dependence. Equally interesting would be the analysis of LIC in menstruating and postmenopausal women.

The lack of liver biopsies as an alternative measure of iron content can also be considered a weakness of this study, but the design of the work a priori excluded such a reference point.

By measuring liver $\mathrm{R} 2 *$ during routine MR testing and referring it to the reference values, patients with mild elevation of ferritin blood levels and no iron overload can avoid invasive diagnostics (e.g., liver biopsy) or invasive blood-letting tests. It should be remembered that an increase in ferritin levels might occur in the course of other disease processes such as hepatitis, obesity, or alcohol consumption ${ }^{45}$.

Also, the MR method used in this study to assess the hepatic iron level has the additional advantage of being able to evaluate the total amount of iron in the whole liver, as opposed to the localized assessment from biopsy, which can cause a significant diagnostic error in the case of an inhomogeneous distribution of iron in the liver ${ }^{46}$.

In summary, MRI R2* has been developed to accurately measure hepatic iron concentration. Our study has shown a normative hepatic R2* value of less than $38 \mathrm{~s}^{-1}$ in a women-dominated (64\%) and relatively large population of healthy young people, which is a valuable reference for the medical community (Fig. 6).

\section{Data availability}

The data that support the findings of this study are available from the corresponding author upon reasonable request.

Received: 15 February 2020; Accepted: 24 September 2020

Published online: 21 October 2020

\section{References}

1. St Pierre, T. G. et al. Noninvasive measurement and imaging of liver iron concentrations using proton magnetic resonance. Blood 105(2), 855-861 (2005).

2. Bacon, B. R., Kwiatkowski, J. L. Approach to the Patient with Suspected Iron Overload. Post TW, ed. UpToDate. Waltham, MA: UpToDate Inc. https://www.uptodate.com. Accessed on 30 Sept 2019.

3. Ghavamzadeh, A., Mirzania, M., Kamalian, N., Sedighi, N. \& Azimi, P. Hepatic iron overload and fibrosis in patients with beta thalassemia major after hematopoietic stem cell transplantation: a pilot study. Int. J. Hematol. Oncol. Stem Cell Res. 9(2), 55-59 (2015).

4. Arthur, M. J. Iron overload and liver fibrosis. J. Gastroenterol. Hepatol. 11(12), 1124-1129 (1996)

5. Piperno, A. Classification and diagnosis of iron overload. Haematologica 83, 447-455 (1998).

6. Wood, J. C. et al. MRI R2 and R2* mapping accurately estimates hepatic iron concentration in transfusion-dependent thalassemia and sickle cell disease patients. Blood 106, 1460-1465 (2005).

7. Strassburg, C. P. \& Manns, M. P. Approaches to liver biopsy techniques-revisited. Semin. Liver Dis. 26, 318-327 (2006).

8. Garcia-Compean, D. \& Cortes, C. Transjugular liver biopsy. An update. Ann. Hepatol. 3, 100-103 (2004). 
9. Yu, H. et al. Multiecho reconstruction for simultaneous water-fat decomposition and T2* estimation. J. Magn. Reson. Imaging 26(4), 1153-1161 (2007).

10. Queiroz-Andrade, M. et al. MR imaging findings of iron overload. RadioGraphics 29, 1575-1589 (2009).

11. Schwenzer, N. F. et al. T2* relaxometry in liver, pancreas, and spleen in a healthy cohort of one hundred twenty-nine subjectscorrelation with age, gender, and serum ferritin. Invest. Radiol. 43(12), 854-860 (2008).

12. Gu, J. et al. Diagnostic value of MRI-PDFF for hepatic steatosis in patients with non-alcoholic fatty liver disease: a meta-analysis. Eur. Radiol. 29, 3564-3573 (2019).

13. Rostoker, G. et al. Histological scores validate the accuracy of hepatic iron load measured by signal intensity ratio and R2* relaxometry MRI in dialysis patients. J. Clin. Med. 9(1), 17 (2020).

14. Alustiza, J. M. et al. Iron overload in the liver diagnostic and quantification. Eur. J. Radiol. 61, 499-506 (2007).

15. Brittenham, G. M. \& Badman, D. G. Noninvasive measurement of iron: report of an NIDDK workshop. Blood 101, 15-19 (2003).

16. Siegelman, E. S., Mitchell, D. G. \& Semelka, R. C. Abdominal iron deposition: metabolism, MR findings, and clinical importance. Radiology 199, 13-22 (1996).

17. Sirlin, C. B. \& Reeder, S. B. Magnetic resonance imaging quantification of liver iron. Magn. Reson. Imaging Clin. N. Am. 18, 359-381 (2010).

18. Princiotto, J. V. \& Zapolski, E. J. Difference between the two iron-binding sites of transferrin. Nature 255, 87-88 (1975).

19. Alexopoulou, E. et al. R2 relaxometry with MRI for the quantification of tissue iron overload in beta-thalassemic patients. J. Magn. Reson. Imaging 23, 163-170 (2006).

20. Brittenham, G. M. et al. Hepatic iron stores and plasma ferritin concentration in patients with sickle cell anemia and thalassemia major. Am. J. Hematol. 42, 81-85 (1993).

21. Hankins, J. S. et al. R2* magnetic resonance imaging of the liver in patients with iron overload. Blood 113(20), 4853-4855 (2009).

22. Karam, L. B. et al. Liver biopsy results in patients with sickle cell disease on chronic transfusions: poor correlation with ferritin levels. Pediatr. Blood Cancer 50, 62-65 (2008).

23. Gandon, Y. et al. Hemochromatosis: diagnosis and quantification of liver iron with gradient-echo MR imaging. Radiology 193, 533-538 (1994).

24. Gandon, Y. et al. Non-invasive assessment of hepatic iron stores by MRI. Lancet 363(9406), 357-362 (2004).

25. Alustiza, J. M. et al. MR quantification of hepatic iron concentration. Radiology 230, 479-484 (2004).

26. Paisant, A. et al. Assessment of liver iron overload by 3 T MRI. Abdom. Radiol. 42(6), 1713-1720 (2017).

27. Anderson, L. J. et al. Cardiovascular T2-star $\left(\mathrm{T} 2^{\star}\right)$ magnetic resonance for the early diagnosis of myocardial iron overload. Eur. Heart J. 22, 2171-2179 (2001).

28. Garbowski, M. W. et al. Biopsy-based calibration of T2* magnetic resonance for estimation of liver iron concentration and comparison with R2 Ferriscan. J. Cardiovasc. Magn. Reson. 16, 40 (2014).

29. Henninger, B. et al. R2* relaxometry for the quantification of hepatic iron overload: biopsy-based calibration and comparison with the literature. Rofo 187, 472-479 (2015).

30. d'Assignies, G. et al. (2018) Non-invasive measurement of liver iron concentration using 3-Tesla magnetic resonance imaging: validation against biopsy. Eur. Radiol. 28(5), 2022-2030 (2018).

31. Wood, J. C. Use of magnetic resonance imaging to monitor iron overload. Hematol. Oncol. Clin. N. Am. 28(4), 747-764 (2014).

32. Acton, R. T. et al. Relationships of serum ferritin, transferrin saturation, and HFE mutations and self-reported diabetes in the Hemochromatosis and Iron Overload Screening (HEIRS) study. Diabetes Care 29, 2084-2089 (2006).

33. Ernst, O. et al. Hepatic iron overload: diagnosis and quantification with MR imaging. Am. J. Roentgenol. 168, 1205-1208 (1997).

34. Papakonstantinou, O. et al. The pancreas in beta-thalassemia major: MR imaging features and correlation with iron stores and glucose disturbances. Eur. Radiol. 17, 1535-1543 (2007).

35. Maris, T. G. et al. Myocardial and liver iron status using a fast $\mathrm{T}^{*} 2$ quantitative MRI ( $\left.{ }^{*} 2 \mathrm{qMRI}\right)$ technique. Magn. Reson. Med. 57, $742-753$ (2007).

36. Argyropoulou, M. I. et al. Liver, bone marrow, pancreas and pituitary gland iron overload in young and adult thalassemic patients: a T2 relaxometry study. Eur. Radiol. 17, 3025-3030 (2007).

37. Chezmar, J. L. et al. Hepatic iron overload: diagnosis and quantification by noninvasive imaging. Gastrointest. Radiol. 15, 27-31 (1990).

38. Obrzut, M. et al. Normative values for magnetic resonance elastography-based liver stiffness in a healthy population. Pol. Arch. Intern. Med. 129(5), 321-326 (2019).

39. Henninger, B., Alustiza, J., Garbowski, M. \& Gandon, Y. Practical guide to quantification of hepatic iron with MRI. Eur. Radiol. 30(1), 383-393 (2020).

40. Nuttall, K. L., Palaty, J. \& Lockitch, G. Reference limits for copper and iron in liver biopsies. Ann. Clin. Lab. Sci. 33, 443-450 (2003).

41. Bassett, M. L., Halliday, J. W. \& Powell, L. W. Value of hepatic iron measurements in early hemochromatosis and determination of the critical iron level associated with fibrosis. Hepatology 6, 24-29 (1986).

42. Serai, S. D. \& Trout, A. T. Can MR elastography be used to measure liver stiffness in patients with iron overload?. Abdom. Radiol. 44(1), 104-109 (2019).

43. Yokoo, T. et al. Quantitative R2* MRI of the liver with rician noise models for evaluation of hepatic iron overload: simulation, phantom, and early clinical experience. JMRI 42(6), 1544-1559 (2015).

44. Tipirneni-Sajja, A. et al. Ultrashort echo time imaging for quantification of hepatic iron overload: Comparison of acquisition and fitting methods via simulations, phantoms, and in vivo data. JMRI 49(5), 1475-1488 (2019).

45. Adams, P. C. et al. HFE mutations in Caucasian participants of the hemochromatosis and iron overload screening study with serum

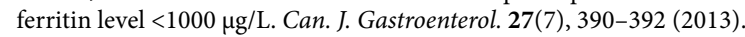

46. Sarigianni, M. et al. Accuracy of magnetic resonance imaging in diagnosis of liver iron overload: a systematic review and metaanalysis. Clin. Gastroenterol. Hepatol. 13(1), 55-63 (2015).

\section{Author contributions}

M.O. initiated and conceived the concept of the study, co-collected the data, co-led the data analysis and interpretation, wrote the first draft of the paper, coordinated draft revisions, and wrote the final manuscript. V.A. co-collected the data, led the statistical analysis, and co-wrote the first draft of the paper. J.Ch. and K.J.G. contributed to the data analysis and interpretation, and draft revisions. R.L.E., B.O., and M.Ch. contributed to data interpretation and draft revisions, K.G. contributed to the study conception, data analysis and interpretation, and draft revisions. All authors read and approved the final version of the manuscript.

\section{Competing interests}

The Mayo Clinic, RLE, KJG and JCh have intellectual property rights and a financial interest in magnetic resonance technology. The remaining authors declare no conflict of interest. 


\section{Additional information}

Correspondence and requests for materials should be addressed to V.A.

Reprints and permissions information is available at www.nature.com/reprints.

Publisher's note Springer Nature remains neutral with regard to jurisdictional claims in published maps and institutional affiliations.

(c) (1) Open Access This article is licensed under a Creative Commons Attribution 4.0 International License, which permits use, sharing, adaptation, distribution and reproduction in any medium or format, as long as you give appropriate credit to the original author(s) and the source, provide a link to the Creative Commons licence, and indicate if changes were made. The images or other third party material in this article are included in the article's Creative Commons licence, unless indicated otherwise in a credit line to the material. If material is not included in the article's Creative Commons licence and your intended use is not permitted by statutory regulation or exceeds the permitted use, you will need to obtain permission directly from the copyright holder. To view a copy of this licence, visit http://creativecommons.org/licenses/by/4.0/.

(c) The Author(s) 2020 